\title{
Nucleotide Sequence of the Salmonella typhimurium mutL Gene Required for Mismatch Repair: Homology of MutL to HexB of Streptococcus pneumoniae and to PMS1 of the Yeast Saccharomyces cerevisiae
}

\author{
JOHN A. MANKOVICH, CHRISTINE A. MCINTYRE, AND GRAHAM C. WALKER* \\ Department of Biology, Massachusetts Institute of Technology, 77 Massachusetts Avenue, \\ Cambridge, Massachusetts 02139
}

Received 6 March 1989/Accepted 1 May 1989

\begin{abstract}
The mutL gene of Salmonella typhimurium LT2 is required for dam-dependent methyl-directed DNA mismatch repair. We have cloned and sequenced the mutL gene of $S$. typhimurium LT2 and compared its sequence with those of the hexB gene product of the gram-positive bacterium Streptococcus pneumoniae and the PMS1 gene product of the yeast Saccharomyces cerevisiae. MutL was found to be quite similar to the HexB mismatch repair protein of $S$. pneumoniae and to the mismatch repair protein PMS1 of the yeast $S$. cerevisiae. The significant similarities among these proteins were confined to their amino-terminal regions and suggest common evolution of the mismatch repair machinery in those organisms. The DNA sequence for mutL predicted a gene encoding a protein of 618 amino acid residues with a molecular weight of 67,761 . The assignment of reading frame was confirmed by the construction of a chimeric protein consisting of the first 30 amino acids of LacZ fused to residues 53 through 618 of MutL. Interestingly, the presence of excess amounts of this fusion protein in wild-type $m u t L^{+}$cells resulted in a trans-dominant effect causing the cell to exhibit a high spontaneous mutation frequency.
\end{abstract}

Accurate duplication of chromosomes is of great importance if a cell line is to guarantee the proper transfer of genetic information from generation to generation. To this end, cells have evolved a number of repair systems that ensure a high level of replication fidelity. One such system (reviewed in references 2,21 , and 30 ) involves the correction of certain mismatched base pairs that have been missed by the proofreading elements of the DNA polymerase replication complex. In Salmonella typhimurium and Escherichia coli, this is accomplished by methyl-directed DNA mismatch repair and is thought to be directed by the transient undermethylation of adenine in the four-base sequence -GATC- of newly replicated DNA. The adenine in the sequence is methylated in the $\mathrm{N}^{6}$ position by the action of the dam methylase (DNA adenine methyltransferase) $(10,15)$. This dam-dependent mismatch repair system requires the products of the mutL, mutS, mutH, and $u v r D$ genes. Mutants that are defective in any of these genes exhibit an elevated spontaneous mutation frequency $(4,34)$. In vitro experiments have shown that hemimethylated -GATC- sites in DNA can direct the repair of specific mismatches in a purified $E$. coli mismatch repair reaction that requires MutL, MutS, MutH, and UvrD (16, 18; P. Modrich, personal communication). In addition, MutL and MutS are involved in very-short-patch repair, which also requires the product of the $\mathrm{dcm}$ gene, DNA cytosine methyltransferase (17). An in vitro cell-free repair system that requires $m u t L, m u t S$, and $\mathrm{dcm}$ gene products has also been developed for very-shortpatch repair (B. Yashar and P. Modrich, personal communication).

Functionally similar repair systems have also been described for the gram-positive organism Streptococcus pneumoniae and for the yeast Saccharomyces cerevisiae. Mu-

* Corresponding author. tants in the hexA or hexB gene of $S$. pneumoniae exhibit an elevated spontaneous mutation frequency $(14,26,38)$. The same is true for the pmsl-1 and pmsl-2 mutants of the yeast $S$. cerevisiae (40). However, these repair systems appear to differ from methyl-directed repair in the method for daughter strand recognition. In $S$. pneumoniae, strand recognition is thought to be directed by breaks in the strand that is to be repaired $(3,14)$. In $S$. cerevisiae, methylation is unlikely to play a role for mismatch repair, since its DNA has neither adenine nor cytosine methylation $(11,28)$.

Previously, we have shown that the mutL, mutS, mutH, and $u v r D$ genes of $S$. typhimurium are functionally analogous to the corresponding $E$. coli genes in that the cloned genes from $S$. typhimurium can fully complement the corresponding $E$. coli mutants both in vivo and in vitro $(18,23-25$; J. Mankovich and G. Walker, unpublished data): We have sequenced the mutS gene of $S$. typhimurium (9), and the mutS gene of $E$. coli has recently been found to be approximately $94 \%$ homologous to the mutS gene of $S$. typhimurium at the amino acid sequence level (Ken Stacey, personal communication). Furthermore, we and Priebe et al. have found that MutS of S. typhimurium and HexA of S. pneumoniae exhibit approximately $38 \%$ overall identity when the amino acid sequences are optimally aligned $(9,27)$. However, these similarities are not distributed evenly throughout the proteins but are grouped in regions with homologies as high as $60 \%$. This finding led us to predict the existence of evolutionarily related repair proteins in other widely divergent organisms, including eucaryotes (9). It is of interest to note that MutL and MutS are involved in a methylationdirected repair process, whereas HexA and HexB functions are independent of the methylation state of the DNA.

A number of in vitro biochemical activities associated with mismatch repair have been determined for the MutS $(23,36)$, MutH (39), and UvrD (helicase II) $(12,22,37)$ proteins. At 
present, the precise role of MutL in the process of mismatch repair is unknown. In this paper, we report the nucleotide sequence for the mutL gene of $S$. typhimurium LT2 and show that the predicted amino acid sequence contains regions of homology to the mismatch repair proteins HexB and PMS1 of $S$. pneumoniae and $S$. cerevisiae, respectively.

\section{MATERIALS AND METHODS}

Bacterial strains, bacteriophage, and plasmids. All work with the $S$. typhimurium mutL gene was performed in $E$. coli, as the mutL gene from $S$. typhimurium is known to fully complement the $E$. coli gene (23). GW5100 (E. coli JM103 [P1 cured]) was used for $\alpha$ complementation of LacZ as well as for production of single-stranded and double-stranded templates for DNA sequencing. E. coli AB1157 (trpA46 $m u t L^{+}$) and its isogenic mutL218::Tn10 derivative were obtained from R. G. Fowler (1) and were used for mutL complementation studies. CSR603 (recAl uvrA6 phr-l) was used in the maxicell experiments (32).

Plasmid pGW1842 (23) was used as the source for the subcloning of the $S$. typhimurium mutL gene. Vectors pBluescript $\mathrm{KS}(+)$ and $\mathrm{SK}(+)$ (Stratagene, La Jolla, Calif.) were used as vectors for cloning and sequencing mutL. Interference-resistant helper phage R408 (Stratagene) was used for single-stranded DNA rescue.

Media. M9, LB, and 2xYT media have been previously described (20). Antibiotics were used in the following concentrations (micrograms per milliliter): ampicillin, 100; tetracycline, 10; nalidixic acid, 40; and rifampin, 100.

In vivo complementation assay. Complementation of mutL218::Tn10 by plasmids carrying various DNA fragments was determined by using a qualitative plate assay. Samples of $100 \mu \mathrm{l}$ from overnight LB broth cultures were plated on nalidixic acid and rifampin plates. Complementation of the mutL218::Tn10 allele by the plasmid resulted in fewer than 5 antibiotic-resistant colonies per plate, whereas failure to complement resulted in more than 100 colonies per plate.

To determine mutation frequencies for specific clones, single colonies were picked and grown in LB broth for $24 \mathrm{~h}$. The cultures were diluted in saline, and approximately 100 to 1,000 cells were inoculated into fresh LB broth, followed by continued incubation for $24 \mathrm{~h}$. The cells were then concentrated 10 -fold in saline, and dilutions were plated on selective medium to determine mutation frequency.

DNA manipulations. Standard methods for analysis of DNA, such as restriction analysis, T4 DNA ligation, blunting of overhanging single-stranded ends, agarose gel electrophoresis, transformation, and plasmid and phage DNA purifications, were performed as described by Maniatis et al. (19).

DNA sequencing. A series of nested deletion mutants was constructed by using the exonuclease III-mung bean nuclease digestion protocol from Stratagene. This allowed isolation of clones containing sequential deletions from a fixed point in the polylinker of the vector to various points in the mutL gene. Any regions of the mutL gene that were not readily accessible for sequencing by the exonuclease IIImung bean nuclease treatment were made so by the removal of specific restriction fragments or by the use of synthetic oligonucleotides to prime chain elongation at specific sites.

Single-stranded and double-stranded DNA sequencing was carried out by the dideoxy-chain termination technique of Sanger et al. (33), using modified T7 DNA polymerase. Analysis of DNA sequence data was carried out using
University of Wisconsin Genetics Computer Group software (5).

Construction and characterization of fusions. Previous restriction mapping of the 2.8-kilobase fragment of pGW1842 placed a unique HindIII site near the predicted amino terminus of the MutL protein (23). By using this restriction site and the BamHI site in the polylinker of the vector, deletions of the amino terminus of MutL were made such that the promoter and amino terminus of the $\alpha$-complementing fragment of $\beta$-galactosidase from the vector was fused to the mutL gene in all three reading frames.

The reading frame I fusion (pGW3403) was prepared by digesting pGW3402 with $B a m \mathrm{HI}$, followed by filling in the 5 -overhanging single-stranded DNA with DNA polymerase I large (Klenow) fragment. This DNA was then further digested with HindIII, followed by removal of the 5 '-overhanging single-stranded DNA with mung bean nuclease. The reading frame II fusion (pGW3404) was prepared by digesting pGW3402 with both BamHI and HindIII, followed by removal of both 5 '-overhanging ends by mung bean nuclease digestion. The reading frame III fusion (pGW3405) was prepared much the same as the reading frame II fusion except that both 5'-overhanging ends were filled in with DNA polymerase I large (Klenow) fragment. Each bluntended DNA mix was then ligated and transformed into a mutL218::Tn10 strain. Representative clones from each transformation mix that had deleted the BamHI-HindIII fragment were chosen for further study.

Identification of plasmid-encoded proteins. Proteins encoded by the mut $^{+}$plasmid (pGW3402) and the reading frame fusion plasmids were analyzed by the maxicell method (32).

\section{RESULTS}

Localization of the mutL gene. The $S$. typhimurium mutL gene was subcloned from plasmid pGW1842 (23) by insertion of the 2.8-kilobase SalI-SmaI restriction fragment into the polylinker region of pBluescript $\mathrm{KS}(+)$ and $\mathrm{SK}(+)$ sequencing vectors. Insertion of the mutL fragment resulted in the mut $L$ gene being placed in both orientations relative to the markers on the vector. Plasmids carrying the mutL-containing restriction fragment in either orientation were found to complement a mutL218::Tn10 strain, causing reduction of the high spontaneous mutation rate to a wild-type level (data not shown).

To localize the mutL gene more precisely, a series of deletion mutants from one end of the cloned 2.8-kilobase fragment was constructed as described in Materials and Methods. These mutants were characterized with respect to the sizes of their deletions and the ability to complement a mutL218::Tn10 strain (Fig. 1A). A subset of these deletions was used for DNA sequence determination.

DNA sequencing. The nucleotide sequence of the $S$. typhimurium LT2 mutL gene was determined (Fig. 2). The sequence was derived from both strands of the DNA spanning the entire region that complemented mutL (Fig. 1A). A single large open reading frame corresponding to the appropriate size for MutL was found (Fig. 1B). This open reading frame was transcribed in the same direction as that predicted by Pang et al. on the basis of the sizes of truncated polypeptides produced by mutL::Tn1000 insertions (23). Fusion protein data supporting this reading frame assignment are described below.

Verification of reading frame choice. Our assignment of the open reading frame was confirmed by analysis of gene 


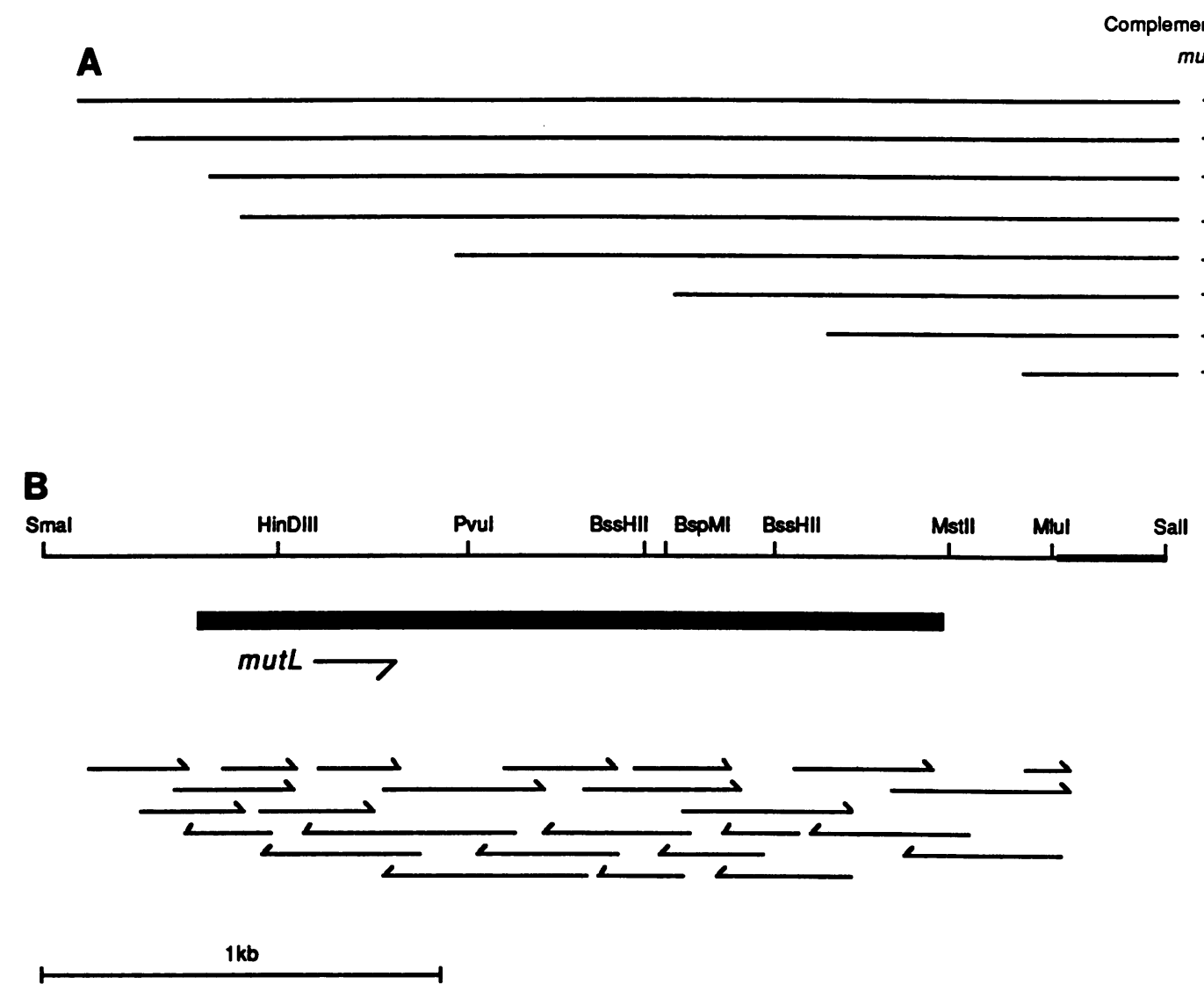

FIG. 1. Partial restriction map of the mutL gene region showing DNA sequencing strategy and location and predicted direction of transcription of the mutL gene. (A) DNA remaining after exonuclease III-mung bean nuclease digestion. + and - , Ability of each subclone to complement mutL218::Tn10. Horizontal arrows in panel B indicate the direction and length of the nucleotide sequence determined for each segment. The darkened area to the right of the MluI site indicates a segment of pBR322 DNA cloned along with the mutL gene from pGW1842. $\mathrm{kb}$, Kilobase.

fusions in all three possible reading frames constructed by replacement of the amino terminus of MutL with that of the promoter and amino terminus of LacZ. These fusions were constructed by using the HindIII site in the mutL gene and the BamHI site in the polylinker of the vector $\mathrm{pSK}(+)$ as described in Materials and Methods.

DNA sequence analysis was performed on three isolates to confirm each reading frame construction (Fig. 3). For reading frame II (pGW3404) and reading frame III (pGW3405), the DNA sequence results were as expected, given the enzyme analysis performed on the DNA fragments (see Materials and Methods). However, for reading frame I (pGW3403) the mung bean nuclease digestion of the HindIII terminus was incomplete, resulting in the removal of only one base instead of four. Since this gave a fusion to the intended reading frame, further screening for the predicted mutant was unnecessary.

The possibility that one of the reading frame fusions would produce a hybrid protein was tested by using the maxicell technique to label plasmid-encoded proteins. Figure $4 \mathrm{de}-$ picts the ${ }^{35} \mathrm{~S}$-labeled proteins encoded by the three reading frame fusion plasmids compared with that of the wild-type mut $^{+}$plasmid. In only one case (frame III, pGW3405) was there a ${ }^{35} \mathrm{~S}$-labeled protein band with an $M_{\mathrm{r}}$ appropriate for the fusion of LacZ with MutL (Fig. 4, lane C). This band was also found to correspond to a very abundant Coomassiestaining protein band that reacted to antibodies directed against MutL and did not appear in either of the other fusions (data not shown). Since this band was not observed in strains with only the chromosomal copy of mutL, the presence of the heavily stained protein band indicated that the fusion protein was in excess to the wild-type MutL protein. This fusion protein consists of the first 30 amino acids of LacZ fused to the predicted amino acid 53 of MutL. As a result, a glutamine residue corresponding to the large open reading frame shown translated in Fig. 2 was created at the fusion junction (Fig. 3).

Since less than $10 \%$ of the MutL protein had been deleted in construction of the frame III fusion, we investigated the possibility that this fusion could complement a mutL strain. The plasmid containing the frame III fusion (pGW3405) was transformed into a mutL218::Tn10 strain, and the spontaneous mutation frequency was determined. The presence of the plasmid had no effect on the mutator phenotype of the mutL218::Tn10 strain. However, elevated spontaneous mutation frequencies were observed when the frame III fusion plasmid (pGW3405) was transformed into a wild-type $m u t L^{+}$ strain. This strain exhibited spontaneous mutation frequencies that were approximately 100 -fold higher than that of wild-type cells containing either the vector or the cloned mut $^{+}$gene (Table 1). It is interesting that the high spontaneous mutation frequencies associated with the frame III fusion in wild-type $m u t L^{+}$cells were approximately five- 


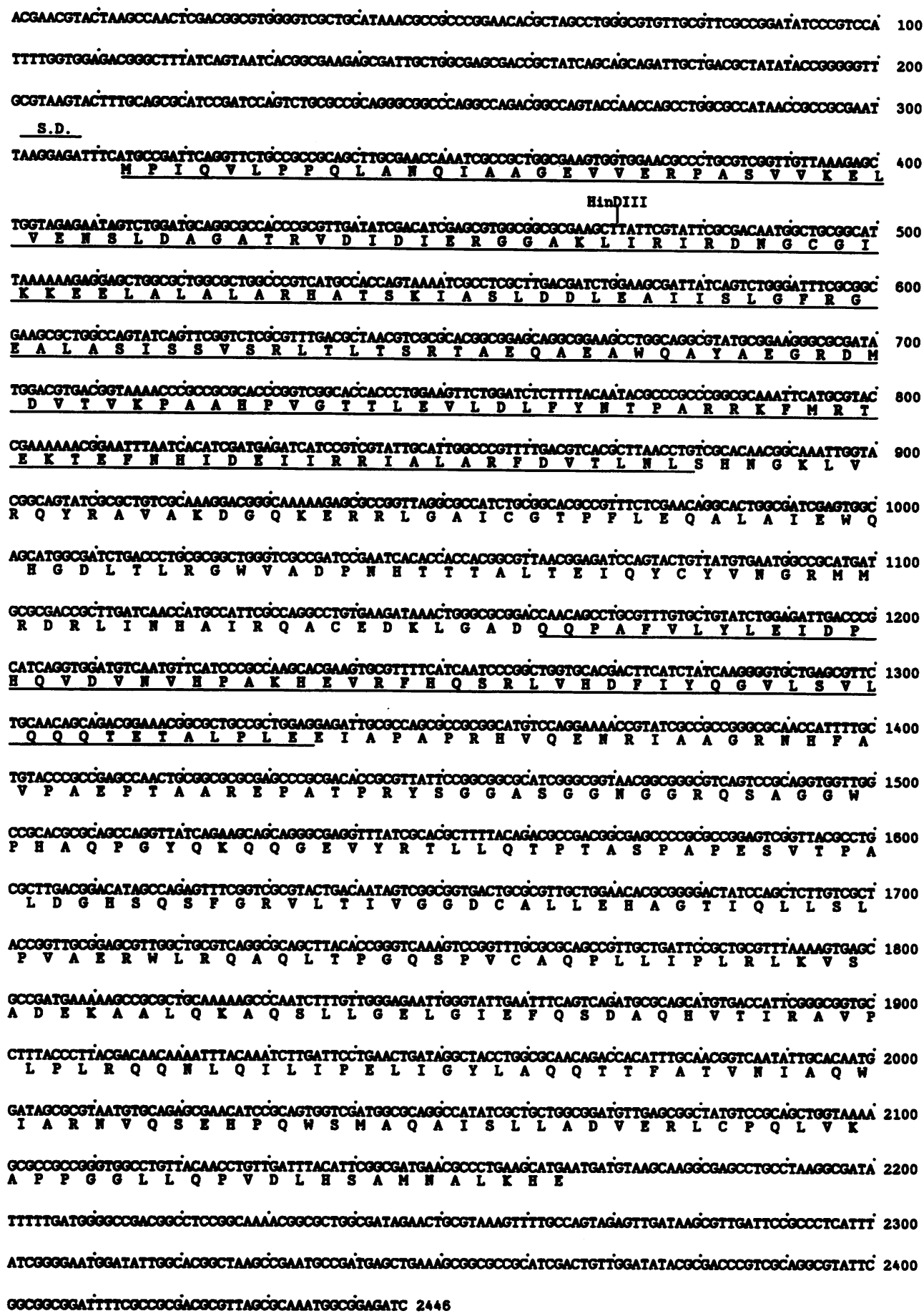

FIG. 2. Nucleotide sequence of mutL gene region from $S$. typhimurium. The derived amino acid sequence is indicated in one-letter code from positions 313 to 2169. The putative ribosome-binding site (S.D.) is indicated proximal to the start codon. The HindIII site used for generating the reading frame fusions is indicated at position 470. Regions of the amino acid sequence that have strongest similarities to HexB and PMS1 are underlined.

fold lower than those observed when the cells were mutL218::Tn10.

Comparison of mutL with mismatch repair genes of $S$. pneumoniae and the yeast $S$. cerevisiae. Previously, we and Priebe et al. reported that the mismatch repair genes mutS of $S$. typhimurium and hexA of $S$. pneumoniae contain regions of significant similarity at the amino acid sequence level $(9$,
27). The impetus for such a comparison was based in part on the similar sizes of the MutS and HexA proteins. Since MutL and HexB have similar $M_{\mathrm{r}} \mathrm{s}(23,26)$, a comparison of the predicted mutL and hexB amino acid sequences was carried out. The nucleotide sequence for the hexB gene of $S$. pneumoniae was determined (29), and a comparison of the predicted amino acid sequences of $m u t L$ and $h e x B$ revealed 


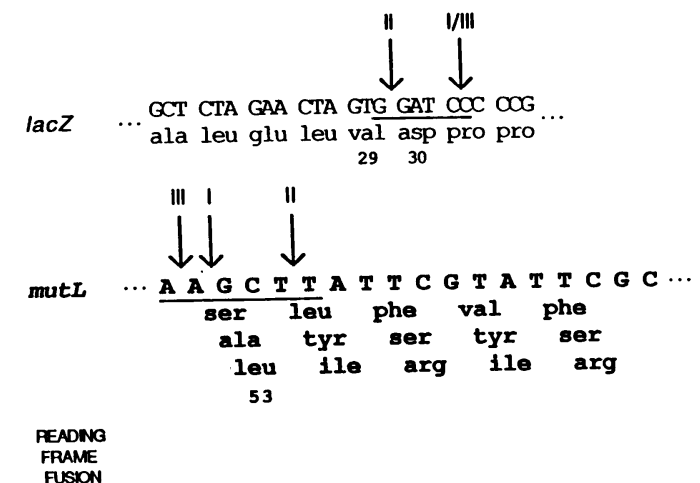

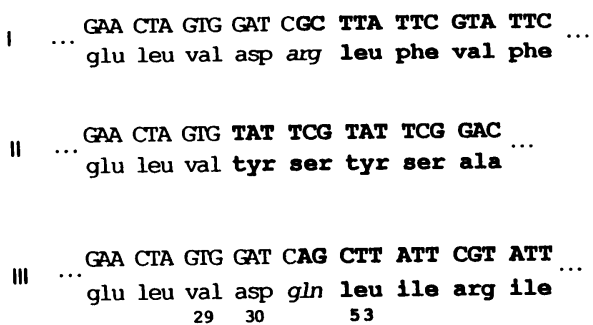

FIG. 3. Construction and partial sequences of reading frame fusions. Shown are DNA and predicted amino acid sequences around the BamHI site in the polylinker of pBluescript $\mathrm{SK}(+)$ and the HindIII site of mutL and the resultant hybrid genes. Arrows indicate the point at which the fusion occurred at each restriction site. Numbers below the amino acids indicate numbers of amino acids from initial methionines of the intact molecules.

significant homologies between the two. The amino-terminal regions of the two putative DNA repair proteins had 99 of $189(52 \%)$ identical amino acids (Fig. 5).

Since mutations in the PMSI gene of the yeast $S$. cerevisiae also result in mutator phenotypes (40), a parallel anal-

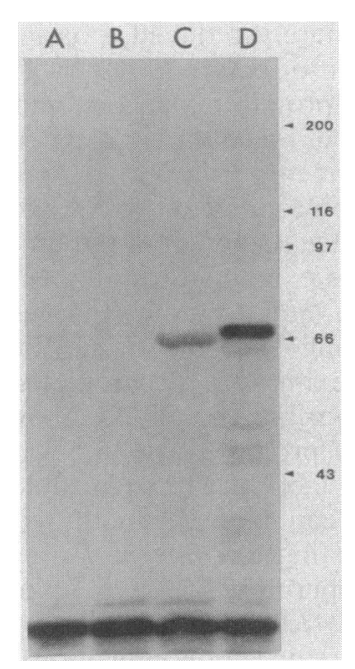

FIG. 4. Plasmid-encoded proteins produced by lacZ-mutL reading frame fusion plasmids and wild-type $m u t L^{+}$pGW3402. The maxicell technique was used to label plasmid-encoded proteins with $\left.{ }^{35} \mathrm{~S}\right]$ methionine. The labeled products were resolved by sodium dodecyl sulfate-electrophoresis through an $8 \%$ polyacrylamide gel and detected by fluorography. Lanes: $\mathrm{A}$ to $\mathrm{C}$, reading frame fusions I (pGW3403), II (pGW3404), and III (pGW3405), respectively; D, wild-type mutL $^{+}$(pGW3402) plasmid-encoded proteins. Molecular weights (in thousands) are shown on the right.
TABLE 1. Mutation frequencies of $m u t L$ and wild-type cells carrying the frame III fusion plasmid

\begin{tabular}{|c|c|c|c|c|}
\hline \multirow{3}{*}{ Plasmid } & \multicolumn{4}{|c|}{$\begin{array}{c}\text { Mutation frequency } \\
\text { (no. of mutants } / 10^{8} \text { viable cells) }{ }^{a}\end{array}$} \\
\hline & \multicolumn{2}{|c|}{ mutL218::Tn 10} & \multicolumn{2}{|c|}{$\mathrm{mutL}^{+}$} \\
\hline & $\mathrm{Nal}^{\mathrm{r}}$ & Rif ${ }^{r}$ & $\mathrm{Nal}^{\mathrm{r}}$ & Rif $^{r}$ \\
\hline $\operatorname{pSK}(+)$ (vector) & 113 & 109 & $<0.10$ & $<0.13$ \\
\hline pGW3402 (mutL $\left.L^{+}\right)$ & $<0.66$ & $<1.28$ & $<0.13$ & $<0.53$ \\
\hline pGW3405 (frame III) & 97 & 340 & 27 & 38 \\
\hline
\end{tabular}

" Determined as described in Materials and Methods. Values are averages of four trials. $\mathrm{Nal}^{\mathrm{r}}$, Spontaneous resistance to nalidixic acid; Rif $^{\mathrm{r}}$, spontaneous resistance to rifampin.

ysis was carried out to compare the deduced sequences of the PMSI (13) and mutL gene products. The amino termini of the proteins had 61 of $190(32 \%)$ of the MutL residues being identical with PMS1, beginning at residue 1 of the predicted MutL protein and residue 31 of the PMS1 protein (Fig. 5). This homology was not as great as the homology seen between MutL and HexB. A similar comparison between HexB and PMS1 showed the same degree of homology as that seen between MutL and PMS1; however, not all of the residues that were identical between MutL and PMS1 corresponded to the same residues that were identical between HexB and PMS1. It is noteworthy that the same regions that were homologous between MutL and $\mathrm{HexB}$ were homologous between MutL and PMS1.

Comparison of all three proteins together showed that they had approximately $24 \%$ of their residues in common within the amino-terminal segment, with 45 of 190 residues being identical among them. These conserved residues were not distributed evenly throughout the amino-terminal sequences but rather were clustered in small segments of the homologous regions. Another segment of approximately 58 residues that contained areas of significant homology existed among the three proteins, starting at residue 284 for MutL, 279 for HexB, and 341 for PMS1 (Fig. 5). We found that $34 \%$

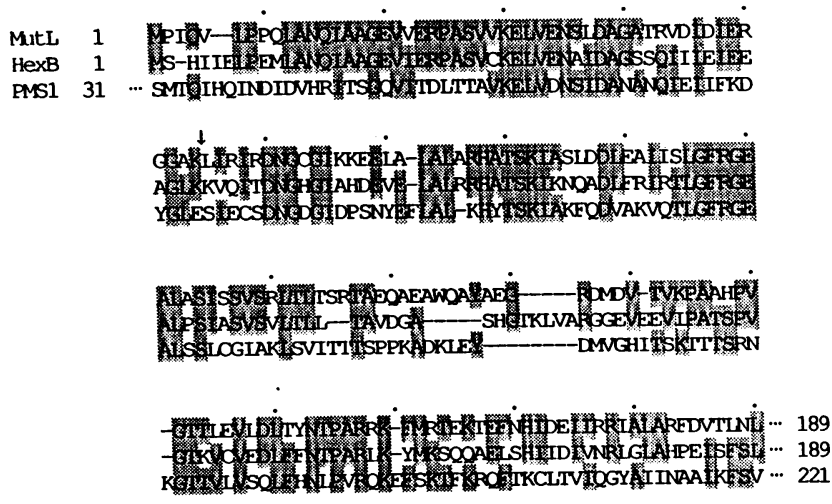

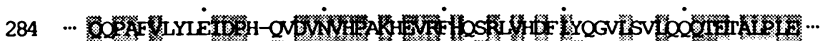

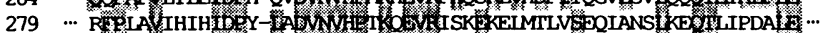

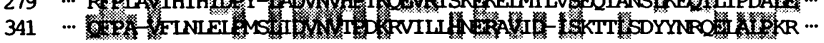

FIG. 5. Homologies of the deduced amino-terminal segments of the MutL, HexB, and PMS1 amino acid sequences. Numbers indicate the position of the corresponding amino acid for each sequence. Identical residues are shaded. Gaps in the sequences have been introduced to maximize the homologies. The point at which the first 30 amino acids of $\mathrm{LacZ}$ were fused to MutL is indicated by the vertical arrow. 
(20 of 58) of the residues of MutL were identical to HexB residues and that $36 \%$ ( 21 of 58 ) were identical to PMS1 residues. The homology between HexB and PMS1 was somewhat less, $22 \%$ (13 of 58 residues). The similarities among all three proteins in this area were clustered in a single region of the segment where $41 \%$ (9 of 22) of the residues were identical.

A third region of homology between HexB and PMS1 was found in the carboxy termini. This region did not correspond to any portion of the MutL protein and is discussed in the accompanying papers $(13,29)$. Additional comparisons of these gene products with the mismatch repair proteins MutS, MutH, and UvrD were carried out, and no significant homologies were observed $(6,7,9)$. Also, a computer search for protein and DNA sequence homologies with mutL by using current releases of the NBRF, EMBL, and GenBank data bases resulted in no significant homologies being found.

\section{DISCUSSION}

In this study, we present the complete nucleotide sequence for the $S$. typhimurium mut $L$ gene, which is required for methyl-directed DNA mismatch repair. Given the amino acid sequence similarities observed between MutS of $S$. typhimurium and HexA of $S$. pneumoniae, we determined the nucleotide sequence for mut $L$ and compared the predicted amino acid sequence with those of other relevant repair proteins now under investigation. On the basis of their similar molecular weights and the mutator phenotypes observed when these genes are defective, we compared MutL of $S$. typhimurium with HexB of $S$. pneumoniae. We also compared the mutL amino acid sequence with the predicted amino acid sequence of the PMSI gene of $S$. cerevisiae; mutations in PMSI also result in a mutator phenotype in yeast. The results of these comparisons revealed that the predicted MutL protein contains regions of significant amino acid sequence homology to the HexB protein of $S$. pneumoniae. Moreover, they show that these same regions have striking similarity to regions in the predicted PMSI gene product of the yeast $S$. cerevisiae. In addition, we have shown that an inactive fusion protein containing greater than $90 \%$ of the MutL protein expressed from a multicopy plasmid can inhibit mismatch repair in a trans-dominant fashion when present in wild-type cells.

The similarities found among MutL, HexB, and PMS1 and those described previously between MutS and HexA reinforce the notion that the DNA mismatch repair machinery evolved very early. There is greater similarity between MutL and HexB than there is between either MutL and PMS1 or HexB and PMS1. This finding may reflect the more recent divergence of the gram-positive and gram-negative bacteria (41). The fact that homologies exist between the procaryotic MutL and HexB proteins and the eucaryotic PMS1 protein suggests a common mode of action for these proteins in the repair of DNA mismatched base pairs. Also, amino acid sequence homologies may exist among various components of mismatch repair in a variety of other procaryotic and eucaryotic organisms.

The mut $L$ DNA sequence of $S$. typhimurium predicts that the gene encodes a protein of 618 amino acid residues with a molecular weight of 67,761 , which is in close agreement with data reported here and previously that mutL from $S$. typhimurium has an $M_{\mathrm{r}}$ of approximately $70,000(23,24)$, as determined by sodium dodecyl sulfate-polyacrylamide gel electrophoresis. The choice of the correct reading frame for the mutL gene was made by creating translational fusions in all three reading frames and analyzing the fusions for the production of an appropriately sized peptide and for the exhibition of an appropriate phenotype. The reading frame choice was based on two observations: (i) only one of the reading frame fusion plasmids produces a plasmid-encoded protein with the correct $M_{\mathrm{r}}$ of the predicted fusion protein, and (ii) the presence of this fusion plasmid results in a partial mutator phenotype in a wild-type ut $^{+}$strain.

Not only does the reading frame fusion experiment allow the correct choice of reading frame for $m u t L$, but it also gives insight as to possible interactions of MutL with the mismatch repair machinery. Greater than $90 \%$ of the MutL amino acid sequence is present in the fusion protein, which consists of the first 30 amino acids of LacZ fused to residues 53 through 618 of MutL. Given the large proportion of MutL in the fusion protein, it is likely that a subset of the MutL domains remains active and could account for the mutator phenotype observed when the fusion is present in wild-type cells.

There are two possible explanations for the apparent dominant negative phenotype observed when the LacZMutL fusion is present in wild-type cells. One possibility is that MutL is capable of regulating repair and that an overabundance of inactive or partially active MutL protein is sufficient to inhibit the production of a component or components of the mismatch repair machinery. This is unlikely given that no evidence for regulation of mut $L$ has yet been found (23; P. Pang and G. Walker, unpublished data). The second possibility is that the trans-dominant effect is due to direct interaction of the LacZ-MutL protein with the less abundant active MutL (MutL dimers have been observed in vitro [8]) or with other components of the mismatch repair machinery. The higher-copy-number inactive fusion protein could be preventing the relatively few copies of active MutL from participating in the repair process. Consistent with this view is the observation that the presence of the fusion protein in $\mathrm{mutL}^{+}$cells results in spontaneous mutation frequencies that are less than that seen in a mutL218::Tn 10 strain. This finding suggests that repair is not completely eliminated but is merely reduced, possibly because of the intracellular dilution of active MutL by the more abundant LacZ-MutL fusion protein. Additional independent support comes from results of recent in vitro experiments suggesting an interaction between purified MutL protein and purified MutS protein on heteroduplexed DNA molecules (8).

The DNA sequence upstream of the predicted start codon AUG for mut $L$ was examined for sequences that are associated with many $E$. coli promoters. A very good match for the ribosome-binding site, the Shine-Dalgarno sequence, was found just proximal to the predicted initiation codon AUG (35). However, no match to known consensus sequences for $E$. coli promoters ( -35 and -10 regions) was obtained (31). The lack of a recognizable promoter in the sequenced region can be explained by results of recent experiments suggesting that the mut $L$ gene of $E$. coli lies within a complex operon between an unknown gene encoding a protein of $M_{\mathrm{r}} 47,000$ and the miaA gene (D. Connolly and $\mathrm{M}$. Winkler, personal communication). It is possible that the transcriptional organization of mutL in S. typhimurium is similar, since we have previously observed that a gene encoding a $47,000-M_{\mathrm{r}}$ protein is located upstream of the mutL gene in $S$. typhimurium (23). Furthermore, our sequencing revealed the presence of an uninterrupted reading frame that is present from the start of the sequenced region to a stop codon UAA just nine base pairs in front of the start codon for the mutL gene of $S$. typhimurium. This reading 
frame is continuous from the beginning of the sequenced region but does not contain a start codon. Presumably, a start codon and promoter for this putative gene could be found by additional DNA sequence determination.

\section{ACKNOWLEDGMENTS}

We thank Prudhomme et al. (29) and Kramer et al. (13) for sharing observations prior to publication. We also thank B. Friedman, L. Haber, and A. Kaufman for constructive comments during preparation of the manuscript.

This work was supported by American Cancer Society grant NP-461E. J.A.M. was a recipient of Public Health Service National Research Service award GM10792 from the National Institutes of Health. C.A.M. was supported in part by the Undergraduate Research Opportunities Program at the Massachusetts Institute of Technology.

\section{LITERATURE CITED}

1. Choy, H., and R. Fowler. 1985. The specificity of base-pair substitution induced by the mutL and mutS mutators in $E$. coli. Mutat. Res. 142:93-97.

2. Claverys, J.-P., and S. Lacks. 1986. Heteroduplex deoxyribonucleic acid base mismatch repair in bacteria. Microbiol. Rev. 50:133-165

3. Claverys, J.-P., V. Mejean, A.-M. Gasc, and M. Sicard. 1983. Mismatch repair in Streptococcus pneumoniae: relationship between base mismatches and transformation efficiencies. Proc. Natl. Acad. Sci USA 80:5956-5960.

4. Cox, E. 1976. Bacterial mutator genes and the control of spontaneous mutation. Annu. Rev. Genet. 10:135-156.

5. Devereux, J., P. Haeberli, and O. Smithies. 1984. A comprehensive set of sequence analysis programs for the VAX. Nucleic Acids Res. 12:387-395.

6. Finch, P., and P. Emmerson. 1984. The nucleotide sequence of the $u v r D$ gene of $E$. coli. Nucleic Acids Res. 12:5789-5799.

7. Grafstrom, R., and R. Hoess. 1987. Nucleotide sequence of the Escherichia coli mutH gene. Nucleic Acids Res. 15:3073-3084.

8. Grilley, M., K. Welsh, S.-S. Su, and P. Modrich. 1989. Isolation and characterization of the Escherichia coli mutL gene product. J. Biol. Chem. 264:1000-1004.

9. Haber, L., P. Pang, D. Sobell, J. Mankovich, and G. Walker. 1988. Nucleotide sequence of the Salmonella typhimurium mutS gene required for mismatch repair: homology of MutS and HexA of Streptococcus pneumoniae. J. Bacteriol. 170:197-202.

10. Hattman, S., J. Brooks, and M. Masurekar. 1978. Sequence specificity of the P1 modification methylase (M $\cdot E c o P 1$ and the DNA methylase (M - Eco dam) controlled by the E. coli dam gene. J. Mol. Biol. 126:367-380.

11. Hattman, S., C. Kenny, L. Berger, and K. Pratt. 1978. Comparative study of DNA methylation in three unicellular eucaryotes. J. Bacteriol. 135:1156-1157.

12. Hickson, I., H. Arthur, D. Bramhill, and P. Emmerson. 1973. The $E$. coli $u v r D$ gene product is DNA helicase II. Mol. Gen. Genet. 190:265-270.

13. Kramer, W., B. Kramer, M. S. Williamson, and S. Fogel. 1989. Cloning and nucleotide sequence of DNA mismatch repair gene PMS1 from Saccharomyces cerevisiae: homology of PMS1 to procaryotic MutL and HexB. J. Bacteriol. 171:5339-5346.

14. Lacks, S., J. Dunn, and B. Greenberg. 1982. Identification of base mismatches recognized by the heteroduplex-DNA-repair system of Streptococcus pneumoniae. Cell 31:327-336.

15. Lacks, S., and B. Greenberg. 1977. Complementary specificity of restriction endonucleases of Diplococcus pneumoniae with respect to DNA methylation. J. Mol. Biol. 114:153-168.

16. Lahue, R., S.-S. Su, and P. Modrich. 1987. Requirement for d(GATC) sequences in Escherichia coli mutHLS mismatch correction. Proc. Natl. Acad. Sci USA 84:1482-1486.

17. Lieb, M. 1987. Bacterial genes mutL, mutS, and dcm participate in repair of mismatches at 5-methylcytosine sites. J. Bacteriol. 169:5241-5246.

18. Lu, A.-L., S. Clark, and P. Modrich. 1983. Methyl-directed repair of DNA base-pair mismatches in vitro. Proc. Natl. Acad.
Sci. USA 80:4639-4643.

19. Maniatis, T., E. F. Fritsch, and J. Sambrook. 1982. Molecular cloning: a laboratory manual. Cold Spring Harbor Laboratory, Cold Spring Harbor, N.Y.

20. Miller, J. 1972. Experiments in molecular genetics. Cold Spring Harbor Laboratory, Cold Spring Harbor, N.Y.

21. Modrich, P. 1987. DNA mismatch correction. Annu. Rev. Biochem. 56:435-466.

22. Oeda, K., T. Horiuchi, and M. Sekiguchi. 1982. The uvrD gene of $E$. coli encodes a DNA-dependent ATPase. Nature (London) 298:98-100.

23. Pang, P., A. Lundberg, and G. Walker. 1985. Identification and characterization of the mutL and mutS gene products of Salmonella typhimurium LT2. J. Bacteriol. 163:1007-1015.

24. Pang, P., S. Tsen, A. Lundberg, and G. Walker. 1984. The mutH, mutL, mutS, and uvrD genes of Salmonella typhimurium LT2. Cold Spring Harbor Symp. Quant. Biol. 49:597-602.

25. Pang, P., and G. Walker. 1983. Identification of the $u v r D$ gene product of Salmonella typhimurium LT2. J. Bacteriol. 153: 1172-1179.

26. Prats, H., B. Martin, and J.-P. Claverys. 1985. The hexB mismatch repair gene of Streptococcus pneumoniae: characterization, cloning, and identification of the product. Mol. Gen. Genet. 200:482-489.

27. Priebe, S., S. Hadi, B. Greenberg, and S. Lacks. 1988. Nucleotide sequence of the hexA gene for DNA mismatch repair in Streptococcus pneumoniae and homology of hexA to mutS of Escherichia coli and Salmonella typhimurium. J. Bacteriol. 170:190-196.

28. Proffitt, J., J. Davie, D. Swinton, and S. Hattman. 1984. 5Methylcytosine is not detectable in Saccharomyces cerevisiae DNA. Mol. Cell. Biol. 4:985-988.

29. Prudhomme, M., B. Martin, V. Mejean, and J.-P. Claverys. 1989. Nucleotide sequence of the Streptococcus pneumoniae hexB mismatch repair gene: homology of HexB to MutL of Salmonella typhimurium and to PMS1 of Saccharomyces cerevisiae. J. Bacteriol. 171:5332-5338.

30. Radman, M., and R. Wagner. 1986. Mismatch repair in Escherichia coli. Annu. Rev. Genet. 20:523-538.

31. Reznikoff, W., D. Siegele, E. Cowing, and C. Gross. 1985. The regulation of transcription initiation in bacteria. Annu. Rev Genet. 19:355-387.

32. Sancar, A., A. Hack, and W. Rupp. 1979. Simple method for identification of plasmid-encoded proteins. J. Bacteriol. 137: 692-693.

33. Sanger, F., S. Nicklen, and A. R. Coulson. 1977. DNA sequencing with chain-termination inhibitors. Proc. Natl. Acad. Sci. USA 74:5463-5467.

34. Shanabruch, W., I. Behlau, and G. Walker. 1981. Spontaneous mutators of Salmonella typhimurium LT2 generated by insertion of transposable elements. J. Bacteriol. 147:827-835.

35. Shine, J., and L. Dalgarno. 1974. The 3'-terminal sequence of Escherichia coli $16 \mathrm{~S}$ ribosomal RNA: complementarity to nonsense triplets and ribosome binding sites. Proc. Natl. Acad. Sci. USA 71:1342-1346.

36. Su, S.-S., and P. Modrich. 1986. Escherichia coli mutS-encoded protein binds to mismatched DNA base pairs. Proc. Natl. Acad. Sci. USA 83:5057-5061.

37. Taucher-Scholtz, G., and H. Hoffman-Berling. 1983. Identification of the gene for DNA helicase II of Escherichia coli. Eur. J. Biochem. 137:573-580.

38. Tiraby, G., and M. Fox. 1972. Marker discrimination in transformation and mutation of pneumococcus. Proc. Natl. Acad. Sci. USA 70:3541-3545.

39. Welsh, K., A.-L. Lu, S. Clark, and P. Modrich. 1987. Isolation and characterization of the Escherichia coli mutH gene product. J. Biol. Chem. 262:15624-15629.

40. Williamson, M., J. Game, and S. Fogel. 1985. Meiotic gene conversion mutants in Saccharomyces cerevisiae. I. Isolation and characterization of PMSI-1 and PMS1-2. Genetics 110:609 646.

41. Woese, C. 1987. Bacterial evolution. Microbiol. Rev. 51:221271. 